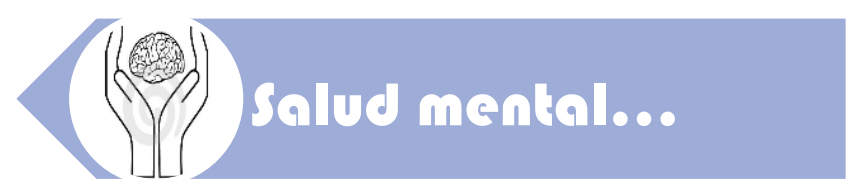

\title{
DETECCIÓN Y NOTIFICACION DEL MALTRATO INFANTIL
}

\author{
Jessica Fernanda Gómez J.+†
}

\section{Resumen}

El maltrato infantil impide el óptimo desarrollo de los niños y las niñas en diversas áreas, por lo que conocer acerca de esta problemática resulta fundamental para la sociedad y, aún más, para quienes trabajan en el área de salud. Este artículo recoge brevemente parte del contexto colombiano sobre los casos de maltrato infantil; asimismo, presenta una revisión de los tipos de maltrato, sus efectos, indicadores y formas de detección y notificación.

Palabras claves: Maltrato a los niños, notificación obligatoria, abuso sexual infantil.

\section{ABSTRACT}

Child abuse make difficult children's development in several areas, so it is essential that not only the society but even the healthcare providers know about this problem. This article summarizes shortly part of the Colombian context of child abuse. In like manner, it presents a review of child abuse types, also its effects, indicators and, the ways to detect and notify it.

Key words: Child abuse, mandatory reporting, child sexual abuse.

\section{EL MALTRATO INFANTIL EN COLOMBIA}

La infancia es una etapa clave en el desarrollo humano, que implica un alto grado de dependencia del niño hacia los adultos para la consolidación de su futuro. La protección de esta población es fundamental para su óptimo desarrollo. Sin embargo, lamentablemente, problemáticas como el maltrato infantil, en Colombia alcanzan cifras que son alarmantes. La presente revisión pretende ser una contribución para los trabajadores de la salud al exponer el debido contexto del problema y explicar sus diferentes modalidades con el propósito de alarmar sobre sus consecuencias, enfatizar en cómo sospecharlo y hacer un llamado para su notificación informando sobre las rutas para hacerlo.

\section{Un problema prevalente}

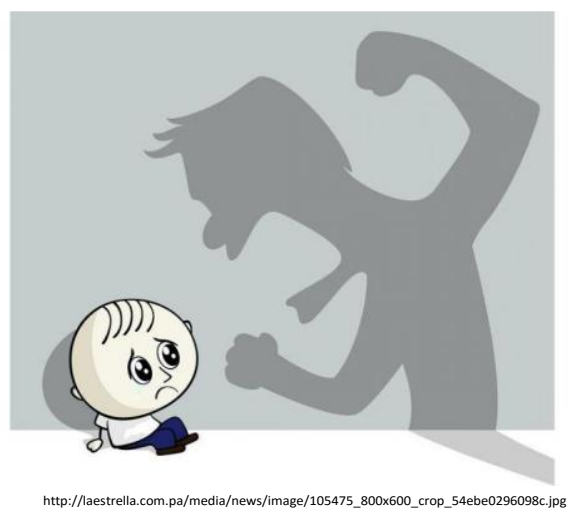

La Defensoría del Pueblo en 2014 advirtió que, entre el 1 de enero y el 4 de abril de ese año, se reportaron 13670 denuncias relacionadas con maltrato infantil (1). Según Forensis, en 2015 se presentaron 10435 casos de violencia física intrafamiliar contra niños, niñas y adolescentes, 33 casos

${ }^{+\dagger}$ Estudiante Maestría en Salud Mental Comunitaria. Universidad El Bosque - jefegoji7@hotmail.com 
más que en 2014 (2). Del total de casos, los padres siguen siendo los principales perpetradores del maltrato con un $63,5 \%$. Las mayores tasas de maltrato se presentan entre los 5 y 14 años.

\section{Desolador}

En cuanto a violencia sexual, según Forensis en 2015 se presentaron 19181 exámenes médico legales por presunto delito sexual entre las edades de 0 a 17 años de edad. Se destaca que, de la población total ( 0 a 80 años y más) a la que se realizó exámenes médico legales por presunto delito sexual, el $75,1 \%$ corresponde a población entre los 0 y 14 años (3). Cifras sumamente preocupantes dadas las enormes implicaciones que tiene este delito en el desarrollo biopsicosocial infantil.

\section{El silencio cómplice}

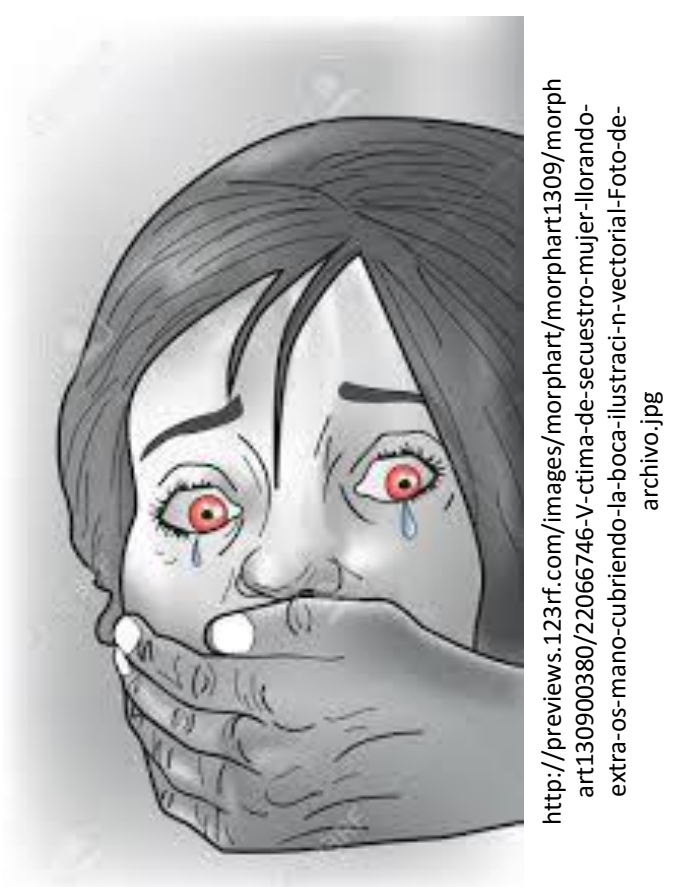

Es necesario destacar que se considera que un inmenso número de casos de maltrato es desconocido por su falta de denuncia; algunos autores aseguran que estos datos conocidos son la punta de un enorme iceberg, de forma que las cifras conocidas podrían constituir solamente un diez o el veinte por ciento de los casos de maltrato existentes (4).

\section{EL MALTRATO INFANTIL Y SUS EFECTOS}

\section{Definición}

El Código de Infancia y Adolescencia define maltrato infantil como "toda forma de perjuicio, castigo, humillación o abuso físico o psicológico, descuido, omisión o trato negligente, malos tratos o explotación sexual, incluidos los actos sexuales abusivos y la violación y, en general, toda forma de violencia o agresión sobre el niño, la niña o el adolescente por parte de sus padres, representantes legales o cualquier otra persona" (5). 


\section{Tipos de maltrato}



Se han descrito los diferentes tipos de maltrato:

Maltrato físico: Es toda agresión manifestada por medio de golpes. Puede tener o no como resultado de lesión física que es producto de castigos únicos o reincidentes, con proporciones y características diferentes. En este tipo de maltrato suelen existir evidencias que facilitan su diagnóstico directo (6).

Maltrato psicológico: Manifestado por insultos, groserías, desprecio, burlas y amenazas para hacer sentir mal a los niños. Incluye también rechazo, aislamiento; ignorar y aterrorizar a los niños (7).

Abandono, negligencia o descuido: Desprotección y falta de cuidado mínimo por parte de las personas que tienen el deber y las condiciones de hacerlo. La negligencia consiste en omisiones que se ven reflejadas de forma inofensiva a lo largo del tiempo; es de difícil detección y fácil de negar (6).

Abuso sexual: Toda actividad sexual entre un niño o niña. Incluye la explotación sexual. Se encuentran evidencias cuando existe penetración, pero, con frecuencia el abuso no es claro y no es posible realizar un diagnóstico directo; en estas situaciones se hace indispensable buscar indicadores indirectos como el relato del menor o el de testigos (8).

\section{Consecuencias}

Son múltiples. En la investigación "Neurobiología del maltrato infantil: el ciclo de la violencia", realizada por Patricia Mesa y colaboradores (9), se aborda desde una revisión y recapitulación de hallazgos de diversas investigaciones de tipo clínico sobre las consecuencias del maltrato infantil sobre el sistema nervioso central. En él se destaca que el maltrato a una edad temprana puede tener consecuencias psicológicas y neurológicas irreversibles. Entre ellas se encuentran cambios a nivel del hipocampo, la amígdala, cuerpo calloso y córtex cerebral. Asimismo, se presentan consecuencias cognitivas, dificultades conductuales y problemas sociales que se asocian son diversas patologías. El maltrato puede provocar en los niños una alta mortalidad y morbilidad, problemas emocionales y comportamientos autodestructivos, así como numerosos déficits cognitivos, entre estos últimos, trastornos del aprendizaje y dificultades académicas. 


\section{Por ende...}

El maltrato infantil reduce la calidad de vida relacionada con la salud física y mental. Los problemas en el desarrollo psicológico y neurobiológico pueden contribuir a psicopatologías a corto y a largo plazo.

\section{Consecuencias}

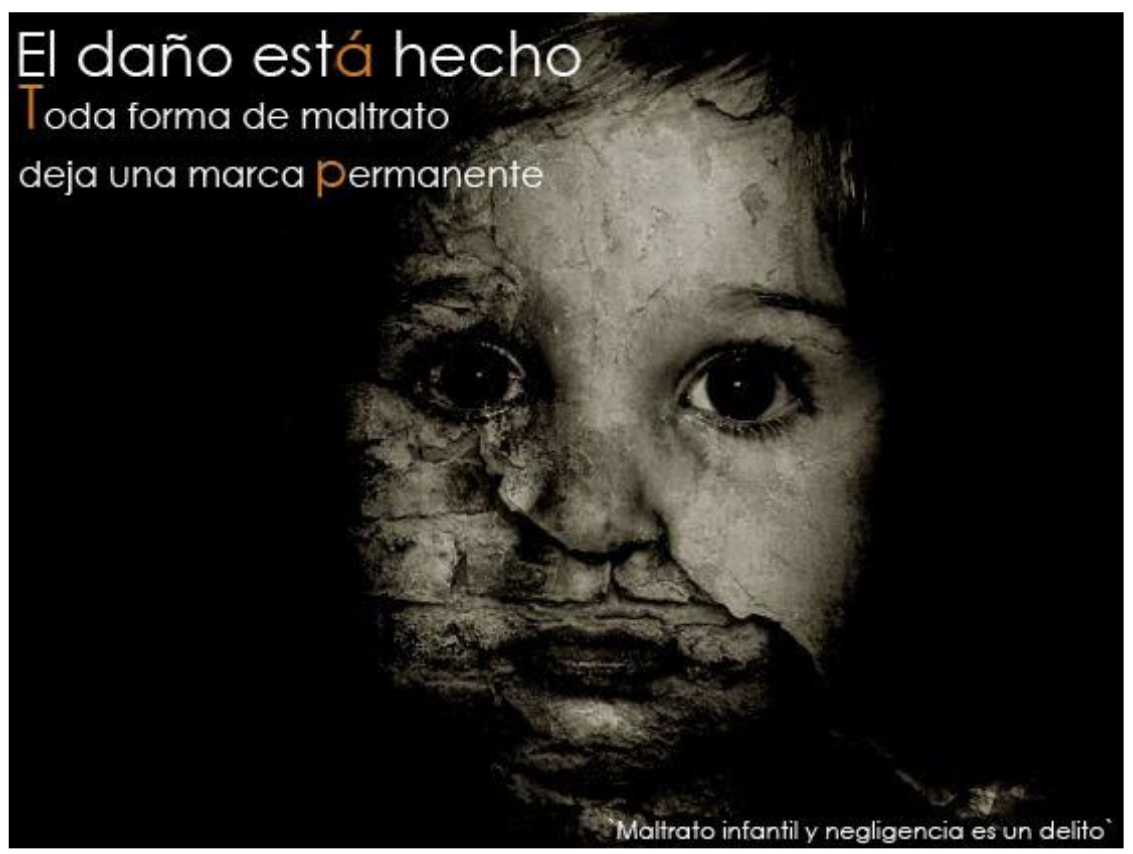

Los trastornos de mayor prevalencia en los niños son la depresión infantil, los problemas de conducta y la delincuencia, los trastornos de conducta antisocial y oposicionista, los trastornos por déficit de atención y los trastornos de estrés postraumático. Además se establecen otras patologías asociadas con el maltrato, como los trastornos de personalidad, la esquizofrenia, el consumo de sustancias psicoactivas, la conducta suicida, la somatización, la ansiedad y la disociación.

\section{Dejando huella}

De acuerdo a estos hallazgos este estudio encuentra que el maltrato infantil, en la mayoría de los casos, genera una interrupción en el desarrollo normal del niño. Esto sustentado en los altos niveles de estrés crónico que provocarán secuelas anatómicas, estructurales y funcionales del cerebro (9).

\section{No olvidar el maltrato emocional}

Por otra parte, frente al maltrato de tipo emocional, Hornor (10) denota que este tipo de maltrato es un problema social grave al que poco se le reconoce en comparación con el maltrato físico y sexual, situación que se explicaría por la dificultad para su identificación y definición, así como por la percepción errónea de que el maltrato infantil de tipo emocional es menos severo que el físico o el sexual, creencia que resulta falsa ya que este tipo de maltrato, al igual que el maltrato físico y el sexual, tienen consecuencias graves y perdurables en los niños. Dada la gran trascendencia que conlleva el maltrato infantil, se hace necesario fortalecer los procesos para su detección y notificación en todos los escenarios, especialmente en los escenarios médicos. 


\section{DETECCIÓN DEL MALTRATO}



La detección del maltrato infantil consiste en identificar una probable situación de maltrato. La detección es un elemento primario para la intervención de los niños y los miembros de la familia que viven dicho fenómeno. Cuando no se realiza, este proceso no se puede dar y no se podrá actuar sobre las causas que dieron lugar al maltrato ni proteger al menor. Es importante que la detección sea lo más precoz posible, de manera que se eviten nefastas consecuencias en el niño e incrementar las probabilidades de éxito en la intervención, tratar las secuelas y revenir la repetición (11).

\section{Buscándolo...}

Se basa en la identificación de indicadores de sospecha. Los indicadores son signos de alarma que indican la posibilidad de maltrato (12). A continuación se mostrarán los indicadores potenciales o activos según el tipo de maltrato, estos indicadores serán divididos en:

Indicadores en el niño:

$\checkmark$ Físicos.

$\checkmark$ Comportamentales.

Indicadores en la conducta del cuidador. 


\section{Indicadores de abuso físico $(8,11)$}

\section{Indicadores en el niño:}

\section{$\checkmark$ Indicadores físicos}

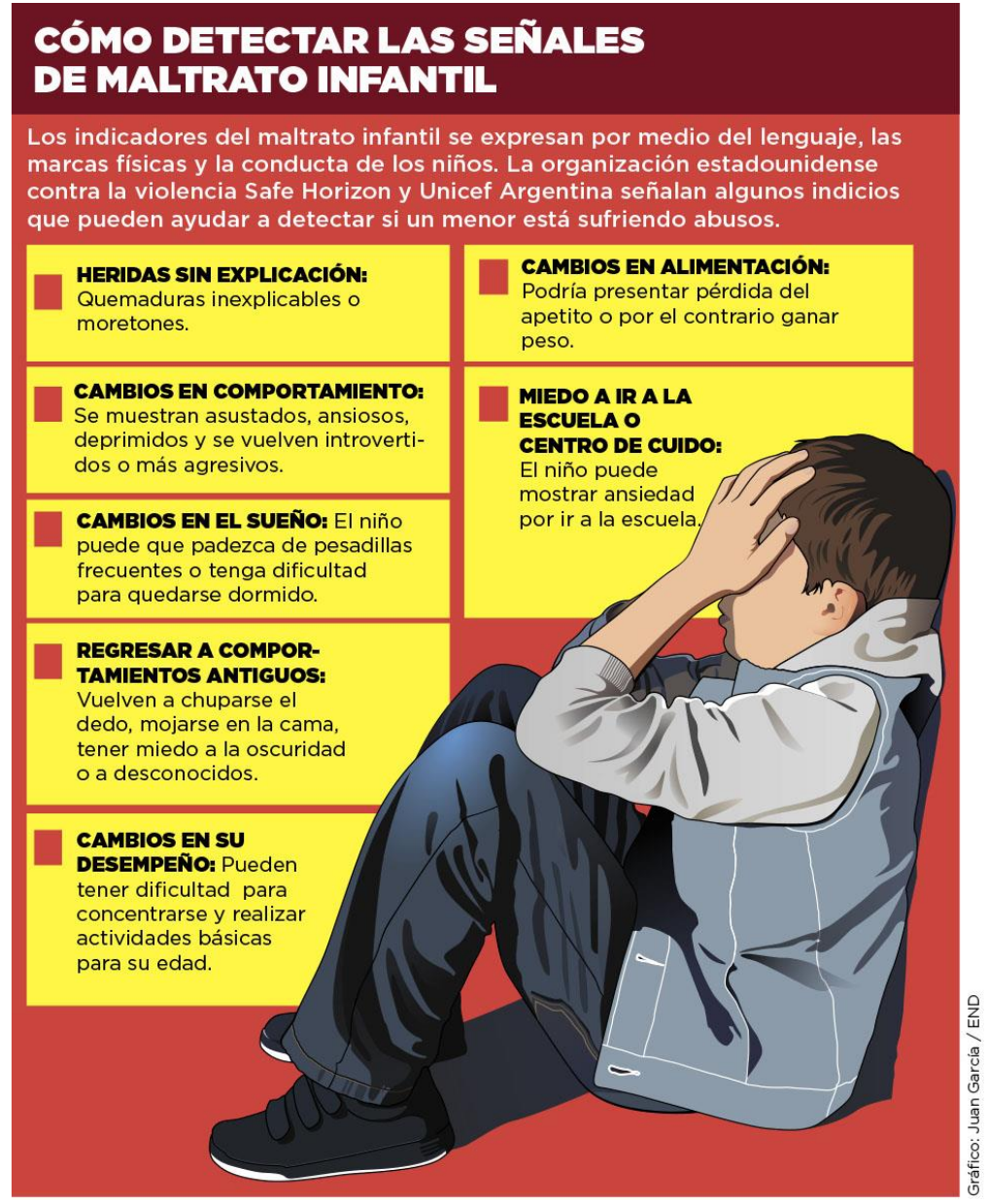

http://endimages.s3.amazonaws.com/news/96f1b7decad011e4be310eb04a1bba78.jpg

- Magulladuras o hematomas: en rostro, labios o boca; en diferentes fases de cicatrización; en zonas extensas del torso, espalda nalgas o muslos; con formas anormales agrupados 0 como señal o marca del objeto con el que ha sido ocasionado; en varias áreas diferentes indicando que el niño ha sido golpeado desde distintas direcciones.

Quemaduras: por cigarros; quemaduras que cubren toda la superficie de las manos (guante) o de los pies (calcetín) o quemaduras en forma de buñuelo en nalgas, genitales; indicativas de inmersión en un líquido caliente; quemaduras en brazos, piernas, cuello o torso provocadas por haber estado atado fuertemente con cuerdas; quemaduras con objetos que dejan señal claramente definida (parrilla, plancha, etc.).

Fracturas: en el cráneo, nariz o mandíbula; fracturas en espiral en los huesos largos (brazos o piernas); en diversas fases de cicatrización; fracturas múltiples; cualquier fractura en un niño menor de dos años. 
- Heridas o raspaduras: En la boca, los labios, encías y ojos, en los genitales externos, en la parte posterior de los brazos, piernas o torso.

- Lesiones abdominales: hinchazón del abdomen, dolor localizado, vómitos constantes.

- Mordedura humana: huella del adulto (separación mayor de $3 \mathrm{~cm}$ entre caninos).

Otros indicadores: Cortes o pinchazos; lesiones internas; asfixia o ahogamiento.

\section{$\checkmark$ Indicadores comportamentales}

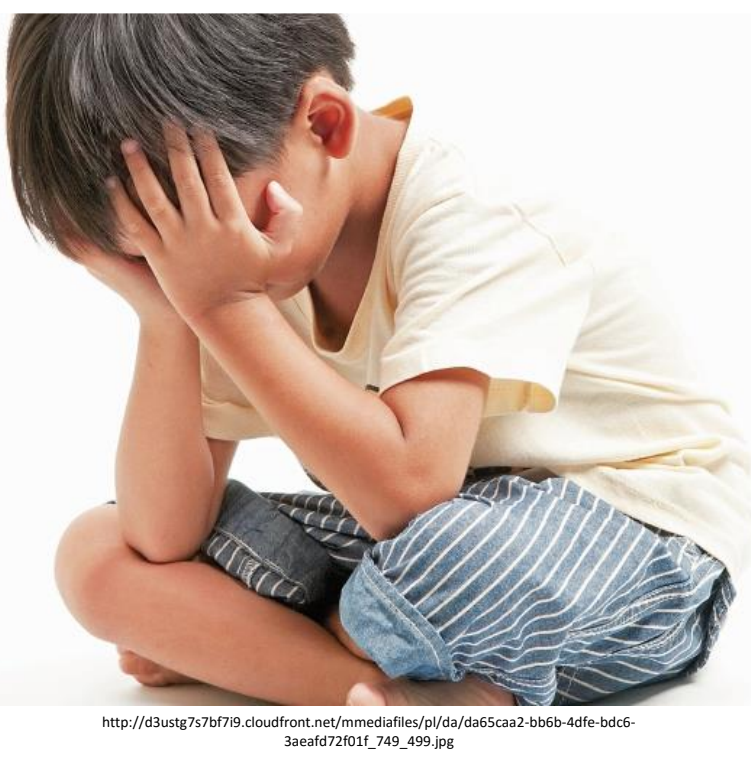

- Cauteloso con respecto al contacto físico con los adultos.

- Se muestra aprensivo cuando otros niños lloran.

- Muestra conductas extremas, por ejemplo, agresividad, o rechazo.

- Parece tener miedo de sus padres o por ir a casa, llora cuando terminan las clases y tiene que irse de la escuela o guardería.

- Dice que su padre/madre le ha causado alguna lesión.

\section{Indicadores en la conducta del cuidador:}

- Fue objeto de maltrato en su infancia.

- Utiliza una disciplina severa, inapropiada para la edad, para la falta cometida y condición del niño.

- No da ninguna explicación con respecto a la lesión del niño o estas son ilógicas, no convincentes o contradictorias.

- Parece no preocuparse por el niño.

- Percibe al niño de manera significativamente negativa, por ejemplo, le ve como malo, perverso.

- Psicótico o psicópata.

- Abusa del alcohol u otras drogas.

- Intenta ocultar la lesión del niño o Proteger la identidad de la persona responsable de este. 


\section{Indicadores de maltrato por abuso sexual (8)(11)}

\section{Indicadores en el niño:}

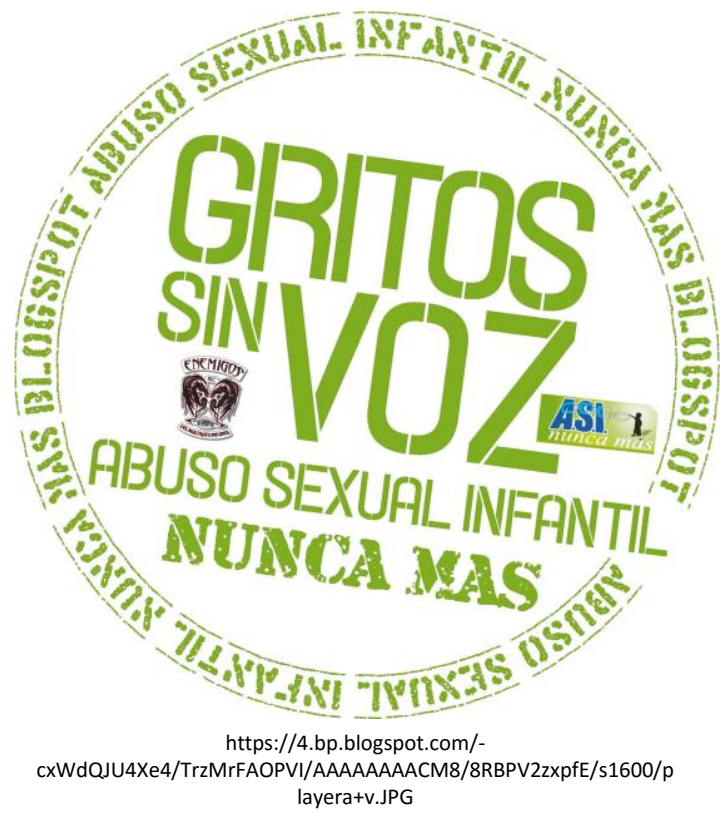

\section{Indicadores físicos:}

- Dificultad para andar y sentarse.

- Ropa interior rasgada, manchada o ensangrentada.

- Se queja de dolor o picor en la zona genital.

- Contusiones o sangrado en los genitales externos, zona vaginal o anal.

- Tiene una enfermedad de transmisión sexual.

- Tiene la cérvix o la vulva hinchados o rojos.

- Tiene semen en la boca, genitales o en la ropa.

- Presencia de cuerpos extraños en uretra, vejiga, vagina o ano.

- Embarazo (especialmente al inicio de la adolescencia).

- Infecciones urinarias de repetición.

\section{$\checkmark$ Indicadores comportamentales}

- Dice que ha sido atacado sexualmente por un padre/cuidador.

- Manifiesta conductas o conocimientos sexuales extraños, sofisticados, inusuales.

- Psicosomáticos.

- Trastornos del sueño y alimentación.

- Diversos: algias abdominales, cefaleas, trastornos neurológicos, respiratorios, esfinterianos, etc., que originan intenso consumo médico sin aclarar las causas.

- Psíquicos: depresiones crónicas, intentos de autolisis, automutilaciones.

- Desvalorización corporal: obesidad, anorexia.

- Problemas de conducta: fugas, fracasos escolares. 
I CARTA COMUNITARIA

Conducta del cuidador

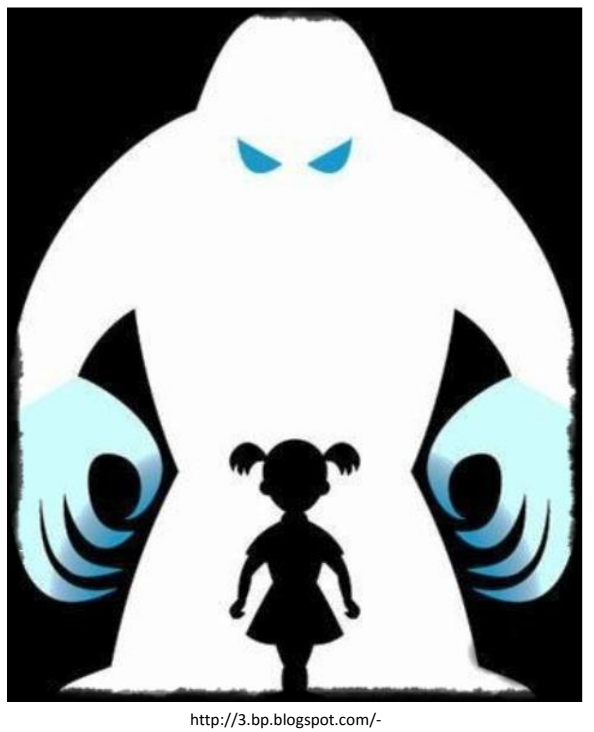

WW1YndVi2fs/UKiwQgXCxj//AAAAAAAAAaU/r69AuaoTM80/s640/sds+(10).jpg

- Extremadamente protector o celoso del niño.

- Alienta al niño a implicarse en actos sexuales o prostitución en presencia del cuidador.

- Sufrió abuso sexual en su infancia.

- Experimenta dificultades en su matrimonio.

- Abusa de las drogas o el alcohol.

- Está frecuentemente ausente del hogar.

\section{Indicadores de abandono, negligencia o descuido $(8,11)$}



Constantemente sucio, escasa higiene, hambriento, inapropiadamente vestido. 
- Constante falta de supervisión, especialmente cuando el niño está realizando actividades peligrosas o durante largos períodos de tiempo.

- Cansancio o apatía permanentes.

- Retraso del crecimiento.

- Infecciones leves, recurrentes o persistentes.

- Consultas frecuentes a los servicios de urgencia por falta seguimiento médico.

- Hematomas inexplicados.

- Accidentes frecuentes por falta de supervisión en situaciones peligrosas.

- Enfermedad crónica llamativa que no genera consulta médica.

- Ropa inadecuada para las condiciones climáticas.

- Alimentación y/o hábitos y horarios inadecuados.

- Ha sido abandonado.

- Retraso en las áreas madurativas.

- Problemas de aprendizaje escolar.

\section{Indicadores de maltrato psicológico}

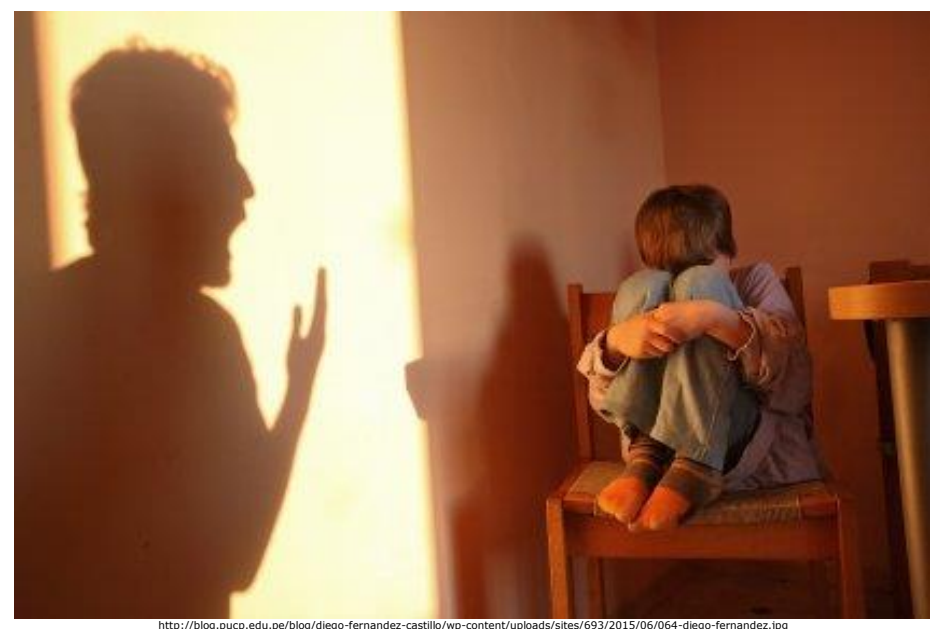

Los síntomas que se pueden presentar en este tipo de maltrato no son exclusivos del mismo y pueden indicar otro tipo de patología. Por tanto, los siguientes síntomas y trastornos son factores de riesgo y no signos inequívocos de la existencia de maltrato psicológico:

- Depresión o intentos de suicidio.

- Trastorno en el control de esfínteres.

- Psicopatías y conductas delictivas.

- Psicosis.

- Labilidad afectiva y trastornos del comportamiento.

- Dificultades y/o retraso en cualquier área del desarrollo evolutivo (psicomotricidad, inteligencia, lenguaje, socialización, etc.).

- Trastornos o enfermedades psicosomáticas a repetición. Alternancia entre agresividadpasividad, dificultad en la esfera de control de impulsos (13). 


\section{DETECCIÓN Y NOTIFICACIÓN EN LOS SERVICIOS DE SALUD EN COLOMBIA}

Bajo la mirada de la corresponsabilidad para la protección de los derechos de la infancia, el Sistema de Seguridad Social en Salud tiene importantes responsabilidades, entre ellas la detección y notificación del maltrato infantil (5).

\section{Lo que debemos saber}

\section{NOTIFICACION AL SIVIM}

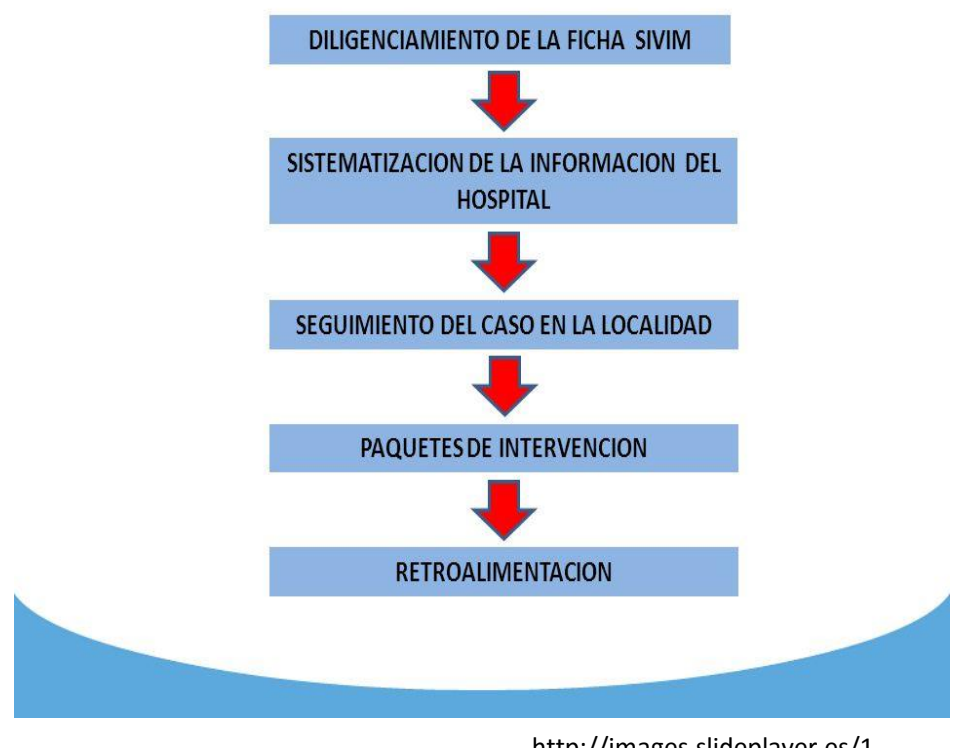

Según la Guía de Atención del Menor Maltratado en Colombia (14), la detección tiene dos aspectos básicos:

1. Búsqueda activa de casos e identificación y recepción del caso. Para la identificación y recepción de casos se destaca que "es importante que el personal de salud tenga en cuenta que en los servicios de urgencias, consulta externa, promoción y prevención, pediatría y ginecología entre otros, se debe explorar permanentemente la presencia de situaciones de violencia intrafamiliar o sexual entre las y los usuarios".

Los aspectos a tener en cuenta en la identificación y recepción de casos son:

1.1. Se recomienda hacer preguntas que indaguen si la persona ha estado relacionada con situaciones de conflicto o violencia.

1.2. El diálogo acerca del tema deberá ser respetuoso, atento y directo y, en un espacio que permita y garantice la privacidad y confidencialidad.

1.3. Es importante que se aclare a la persona la necesidad de seguir procesos de notificación y los límites de la confidencialidad.

1.4. En caso de atender a comunidades indígenas, es importante tener presente que la población indígena cuenta con su propio sistema legislativo. Sin embargo, no se debe olvidar que en la Convención Internacional de los Derechos de los Niños, los derechos de niños y niñas tienen prelación sobre los demás (incluidos los culturales). 


\section{El abordaje}

Las siguientes son las recomendaciones para la detección y la atención de violencia en general, según la Guía de Atención del Menor Maltratado (14):

$\checkmark \quad$ El personal de salud deberá estar atento a cualquier indicio de violencia: iSospechar e indagar!

$\checkmark \quad$ No culpar o responsabilizar a la víctima sobre su situación de violencia.

$\checkmark \quad$ La evaluación deberá realizarse con respeto, sensibilidad, calidez y evitando sesgos y juicios.

$\checkmark \quad$ Si el personal sanitario tiene dudas de que la persona es víctima, se deberá documentar en la historia clínica lo que se evaluó al respecto, la reacción del paciente, detallando el impacto en la salud.

$\checkmark \quad$ Cuando el paciente reporte algún tipo de maltrato o abuso se deberá describir y brindar información, orientación y remisión. Todas estas acciones deberán quedar consignadas.

\section{Acciones para la detección del maltrato infantil (11)}

\begin{tabular}{|c|}
\hline $\begin{array}{l}\text { IDENTIFICAR LOS CASOS SOSPECHOSOS DE } \\
\text { MALOS TRATOS }\end{array}$ \\
\hline $\begin{array}{l}\text { - Estar atento a signos que puedan indicar maltrato o } \\
\text { negligencia. } \\
\text { - Informar sobre sospechas a las autoridades } \\
\text { competentes. } \\
\text { - Llevar registros objetivos del estado físico del niño, } \\
\text { su conducta con los padres, otras personas y el } \\
\text { medio ambiente y, de las entrevistas con los } \\
\text { miembros de las familias. }\end{array}$ \\
\hline EVALUAR LA AMPLITUD DE LAS LESIONES \\
\hline $\begin{array}{l}\text { - Realizar la evaluación física. } \\
\text { - Evaluar el estado emocional y la conducta. } \\
\text { - Participar en las pruebas diagnósticas. }\end{array}$ \\
\hline
\end{tabular}

\section{Notificación del maltrato infantil}

2. Notificar es, según lo define el Protocolo de Intervención Sanitaria en casos de Maltrato Infantil (12), "transmitir o trasladar información sobre el supuesto caso de riesgo, de sospecha o de maltrato infantil comprobado, sobre su familia y sobre el propio informante. Es una condición necesaria para posibilitar la intervención para mejorar las condiciones de las personas menores y su familia y una obligación legal y ética del profesional. La notificación ha de hacerse ante la sospecha de cualquier situación de desprotección o maltrato, no es necesaria la certeza".

\section{Activar las rutas}

La notificación del maltrato infantil permitirá calcular indicadores de incidencia, prevalencia y reincidencia del maltrato; asimismo, permitirá activar la ruta de manejo intersectorial y/o especializado de los niños víctimas violencia. Cabe destacar que se debe notificar los casos sospechosos o probables de maltrato y, resaltar que la ficha de notificación es un medio y no un fin, pues más allá de la visibilización del maltrato, es importante el seguimiento y la implementación de la ruta de atención, ya que la atención y la vigilancia no son procesos separados (15). 
La periodicidad de la notificación del maltrato es semanal e inmediata. La notificación inmediata se refiere a todo caso de violencia sexual y a aquellos casos que revisten riesgo de fatalidad. Al realizarse la valoración es necesario considerar el riesgo de repetición de la agresión hacia la víctima y no solo la severidad del evento violento. Estos casos se deben notificar dentro de las 24 horas siguientes de haberse captado la información, facilitando su denuncia y realizando la gestión encaminada hacia la atención integral del mismo así como a las acciones de las autoridades competentes (16).

\section{Los formatos}

\section{Ruta del Paciente con Maltrato - Esquema I}

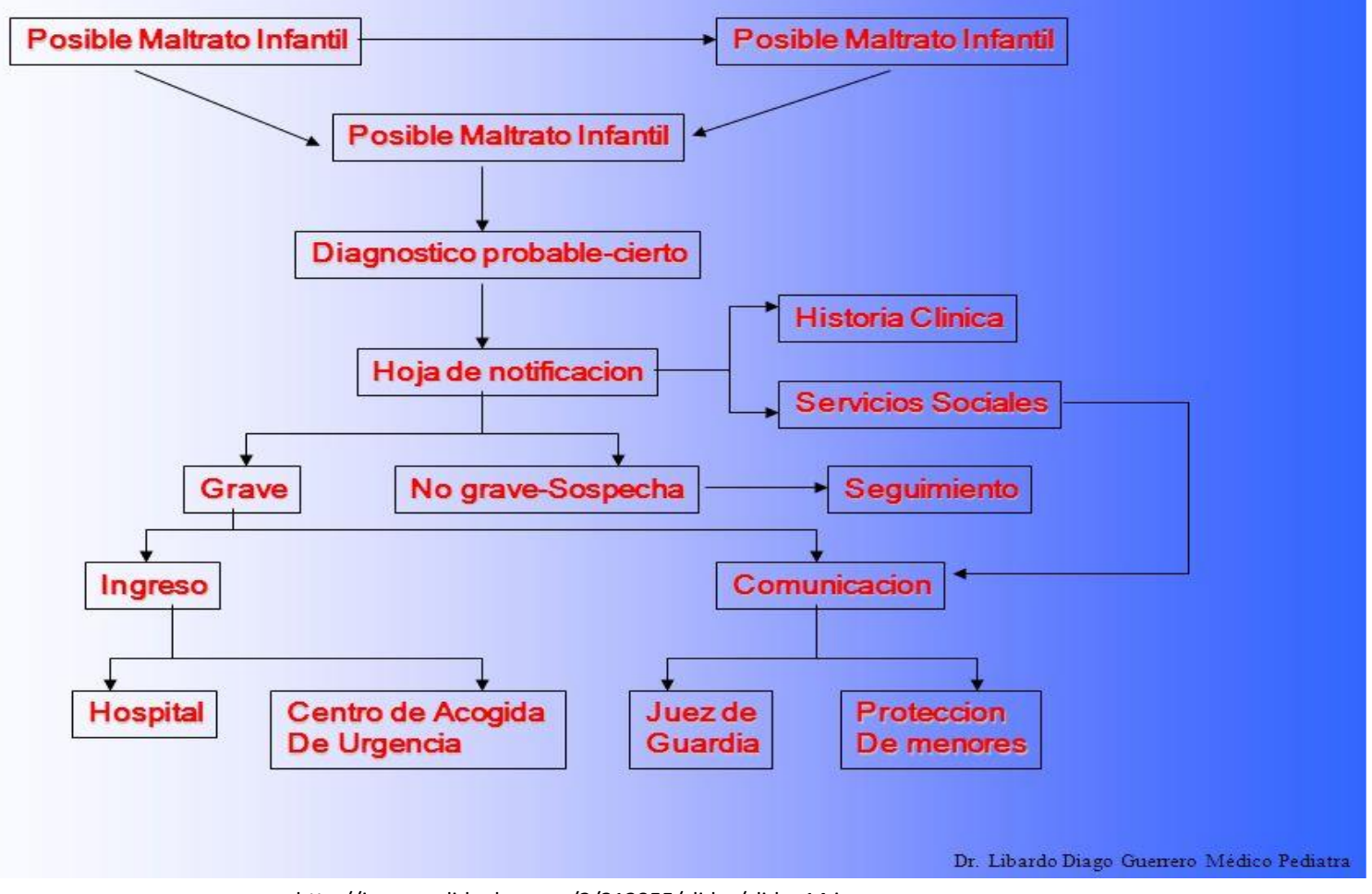

http://images.slideplayer.es/2/312955/slides/slide_14.jpg

Los pasos dispuestos para notificar el maltrato son los siguientes:

$\checkmark \quad$ Registrar en la historia clínica.

$\checkmark \quad$ Llenar la ficha de notificación al sistema de vigilancia epidemiológica.

$\checkmark$ Remitir la ficha a la secretaria de salud municipal o departamental según sea el caso (17).

Cada institución de salud deberá tener su propio protocolo para la atención de casos de maltrato infantil, el cual debe ser conocido por sus trabajadores. 


\section{CONCLUSIONES}

El maltrato infantil es un factor de riesgo para el desarrollo de los niños en el área social, emocional, física y biológica e implica profundas cicatrices para la sociedad. Por esta razón es necesario prevenir, identificar y notificar el maltrato, ya que por medio de estas acciones se protegen los derechos de los niños colombianos. Los profesionales en salud tienen un papel fundamental en la protección de los menores.

El conocer los signos de maltrato infantil es fundamental para la detección del maltrato y posterior notificación. La notificación no solo permite conocer de manera más clara la problemática sino, principalmente, una atención adecuada a los y las menores víctimas de maltrato infantil.

\section{REFERENCIAS}

(1) Defensoría del Pueblo. Alarma por incremento del maltrato infantil en Colombia. [Internet]. [citado 3 de febrero de 2016]. Disponible en: http://defensoria.gov.co/es/nube/noticias/1114/Alarma-por-incremento-del-maltrato-infantil-enColombia-conflicto-armado-Defensor\%C3\%ADa-del-Pueblo-maltrato-infantil-D\%C3\%ADa-del-Ni\%C3\%B1o.htm

(2) Acosta M. Comportamiento de la violencia intrafamiliar [Internet]. Colombia, 2015 [citado 31 de octubre de 2016]. Disponible en: http://www.medicinalegal.gov.co/documents/88730/3418907/4.+VIOLENCIA+INTRAFAMILIAR$+1+$ parte.pdf/57a54b37-954e-4c84-8fec-ab2566a592b6

(3) Cifuentes SL. Exámenes médico legales por presuntos delito sexual. [Internet]. Colombia, 2015 [citado 2 de noviembre de 2016]. Disponible en:

http://www.medicinalegal.gov.co/documents/88730/3418907/6.+VIOLENCIA+SEXUAL.pdf/6c8bff03-1ec8-4c24-955a$393 e 28255398$

(4) Sanmartín J. Violencia contra niños. 4ta ed. España: Ariel; 2008.

(5) Leyes desde 1992 - Vigencia expresa y control de constitucionalidad [LEY_1098_2006] [Internet]. [citado 3 de febrero de 2016]. Disponible en: http://www.secretariasenado.gov.co/senado/basedoc/ley_1098_2006.html

(6) UNICEF. Maltrato Infantil en Chile [Internet]. 2005 [citado 3 de febrero de 2016]. Disponible en: http://unicef.cl/web/unicef-responde-maltrato-infantil-en-chile/

(7) www.altas-buscadores.com, Comunicaciones IOA de. ICBF para niños, niñas y adolescentes - ICBF Colombia [Internet]. [citado 3 de febrero de 2016]. Disponible en:

http://www.icbf.gov.co/portal/page/portal/ICBFNinos/MitosYVerdades/Violencia/MaltratoInfantil

(8) Gómez R, Hernández B, Rojas U, Santacruz O, Uribe R. Psiquiatría clinica: Diágnostico y tratamiento en niño, adolescentes y adultos. 3ra ed. Bogotá: Editorial Médica Internacional; 2008.

(9) Moya L. Neurobiología del maltrato infantil: el «ciclo de la violencia». 2011; 52(8):489-503.

(10) Hornor G. Emotional maltreatment. J Pediatr Health Care off Publ Natl Assoc Pediatr Nurse Assoc Pract. 2012; 26(6):436-42.

(11) Díaz HJ, Casado J, García GE, Ruiz DM, Gómez J. Atención de enfermería al maltrato infatil. España: Instituto Madrileño del menor y la familia.

(12) Protocolo de intervención sanitaria en casos de maltrato infantil [Internet]. Salud y Políticas sociales. [citado 3 de febrero de 2016]. Disponible en: http://www.redsabia.org/file.php?file=\%2F1\%2FProtocolo_Maltrato_Infantil_v8_DEFINITIVO_PUBLICADO.pdf

(13) Díaz JA, García E, Casado J, Ruiz MA. Atención al maltrato infantil désde los servicios sociales. 12/01. España: Instituto Madrileño del menor y la familia.

(14) Ministerio de Salud. Guía de atención al Menor Maltratado [Internet]. [citado 3 de febrero de 2016]. Disponible en: http://www.saludpereira.gov.co/attachments/article/96/Guia\%20de\%20atencion\%20al\%20MenorMaltratado.pdf

(15) Forero LJ. Vigilancia en salud pública de la violencia contra la mujer, violencia intrafamiliar y violencia sexual. [Internet]. instituto Nacional de Salud [citado 16 de noviembre de 2016]. Disponible en: http://www.ins.gov.co/Noticias/Memorias\%20Reunin\%20Nacional\%20de\%20Vigilancia\%20y\%20Control\%20e/1-3violencia.pdf

(16) Salud Capital [Internet]. Vigilancia en salud pública. Violencia Intrafamiliar [citado 16 de noviembre de 2016]. Disponible en: http://www.saludcapital.gov.co/sitios/VigilanciaSaludPublica/Paginas/ViolenciaIntrafamiliar.aspx

(17) Ministerio de Salud y Protección Social [Internet]. Definiciones básicas [citado 16 de noviembre de 2016]. Disponible en: https://www.minsalud.gov.co/Documentos\%20y\%20Publicaciones/FICHERO\%20PAGS\%20INTERNAS.pdf



